\title{
Strong sum distance in fuzzy graphs
}

\author{
Mini Tom ${ }^{1 *}$ and Muraleedharan Shetty Sunitha ${ }^{2}$
}

\begin{abstract}
In this paper the idea of strong sum distance which is a metric, in a fuzzy graph is introduced. Based on this metric the concepts of eccentricity, radius, diameter, center and self centered fuzzy graphs are studied. Some properties of eccentric nodes, peripheral nodes and central nodes are obtained. A characterisation of self centered complete fuzzy graph is obtained and conditions under which a fuzzy cycle is self centered are established. We have proved that based on this metric, an eccentric node of a fuzzy tree $G$ is a fuzzy end node of $G$ and a node is an eccentric node of a fuzzy tree if and only if it is a peripheral node of $G$ and the center of a fuzzy tree consists of either one or two neighboring nodes. The concepts of boundary nodes and interior nodes in a fuzzy graph based on strong sum distance are introduced. Some properties of boundary nodes, interior nodes and complete nodes are studied.
\end{abstract}

Keywords: Fuzzy graph; Strong sum distance; Fuzzy cycle; Fuzzy tree; Boundary; Interior

\section{Introduction}

The theory of fuzzy graphs was developed by Rosenfeld (1975) in the year 1975. During the same time Yeh and Bang (1975) have also introduced various connectedness concepts in fuzzy graphs. Rosenfled (1975) introduced the concept of $\mu$-distance in fuzzy graphs. The author has defined $\mu$ - length of any $u-v$ path $P$ as the sum of reciprocals of arc weights in $P$ and distance between $u$ and $v$ called the $\mu$-distance denoted by $d_{\mu}(u, v)$, as the the smallest $\mu$ - length of $P$. In a fuzzy graph $G$ : $(V, \sigma, \mu), d_{\mu}(u, v)$ is a metric on $V \forall u, v \in V$. Based on this $\mu$-distance Bhattacharya (1987) has introduced the concepts of eccentricity and center in fuzzy graphs and the properties of this metric are further studied by Sunitha and Vijyakumar (1998). The geodetic iteration number and geodetic number of fuzzy graphs based on $\mu$-distance was introduced by Linda and Sunitha (2013). Abdul Jabbar et al. (2009) introduced the concept of fuzzy planar graph and discussed some of its interesting properties. Recently, Pal et al. (2013) and Samanta et al. (2014) introduced and investigated the concept of fuzzy planar graphs and studied several properties. Noura and Akram (2014) studied isomorphism between intuitionistic fuzzy planar graphs. Interval valued fuzzy planar graphs and interval valued fuzzy dual graph are defined by Tarasankar

\footnotetext{
*Correspondence: minitom2001@yahoo.com

${ }^{1}$ Department of Mathematics, SCMS School Of Engineering and Technology, Karukutty, 683582 Kerala, India

Full list of author information is available at the end of the article
}

et al. (2014). Some properties of interval valued fuzzy planar graphs and interval valued fuzzy dual graph are also studied by the authors. Talebi and Rashmanlou (2013) studied isomorphism on interval valued fuzzy graph. Rashmanlou and Pal (2013) defined isometry on interval valued fuzzy graphs and established that isometry on interval valued fuzzy graphs is an equivalence relation. The same authors (Pal and Rashmanlou 2013) also defined irregular interval valued fuzzy graphs and their various classifications. Recently, Akram et al. introduced the concepts of bipolar fuzzy graphs and interval-valued fuzzy line graphs (Akram 2011, 2012, 2013; Akram and Dudek 2011, 2012). Further the author has defined length, distance, eccentricity, radius and diameter of a bipolar fuzzy graph and has introduced the concept of self centered bipolar fuzzy graphs (Akram and Karunambigai 2011). Namboothiri et al. (2013) discussed Cayley fuzzy graphs. Alshehri and Akram (2013) introduced the concept of Cayley bipolar fuzzy graphs and investigated some of their properties. The author has also introduced the concept of an antipodal intuitionistic fuzzy graph and self median intuitionistic fuzzy graph of the given intuitionistic fuzzy graph (Akram and Karunambigai 2012). Akram and Alshehri (2014) introduced various types of intuitionistic fuzzy bridges, intuitionistic fuzzy cut vertices, intuitionistic fuzzy cycles and intuitionistic fuzzy trees in intuitionistic fuzzy graphs and investigated some of their interesting properties. To model ecological problems, in 1968 Cohen (1968) introduced the notion of

\section{是 Springer}

(c) 2015 Tom et al. This is an Open Access article distributed under the terms of the Creative Commons Attribution License (http://creativecommons.org/licenses/by/4.0), which permits unrestricted use, distribution, and reproduction in any medium, provided the original work is properly credited. 
competition graphs. Fuzzy competition graph was introduced by Samanta and Pal (2013). Two generalizations of fuzzy competition graph as fuzzy $k$-competition graphs and $p$-competition fuzzy graphs are also defined by the same authors. In Samanta et al. (2015) define another generalization of fuzzy competition graph, called $m$-step competition graph. Bhutani and Rosenfeld have introduced the concepts of strong arcs (Bhutani and Rosenfeld 2003a), fuzzy end nodes (Bhutani and Rosenfeld 2003c) and $g$-distance in fuzzy graphs (Bhutani and Rosenfeld 2003b). The geodesic eccentricity and geodesic center of a fuzzy graph $G$ is also discussed in (Bhutani and Rosenfeld 2003b). Further studies based on the $g$-distance are carried out by Sameena and Sunitha (2008) and (Sameena and Sunitha 2011). The concepts of $g$-peripheral nodes, $g$-boundary nodes and $g$-interior nodes based on $g$-distance was introduced by Linda and Sunitha (2012). Nagoorgani and Umamaheswari introduced the concept of fuzzy detour $\mu$-distance (Nagoorgani and Umamaheswari 2010). The authors further defined fuzzy detour $\mu$-center and studied its properties. Fuzzy detour $g$-distance was introduced by Linda and Sunitha (2014a) and in (Linda and Sunitha 2014b), the authors introduced fuzzy detour $g$ - boundary nodes and fuzzy detour $g$ - interior nodes in fuzzy graphs. In this paper we introduce the concept of strong sum distance in fuzzy graphs and a study on boundary nodes and interior nodes of a fuzzy graph based on this distance is carried out.

Section 2 contains preliminaries and in section 3, strong sum distance in fuzzy graphs is defined and proved that it is a metric. Based on this metric, eccentricity, radius, diameter, center in fuzzy graphs are defined. Necessary conditions for a fuzzy graph to be self centered are obtained in this section. By an example it is shown that a unique eccentric node fuzzy graph with each node eccentric need not be self centered. Sufficient conditions for a fuzzy cycle to be self centered is given in section 4. A necessary and sufficient condition for a complete fuzzy graph to be self centered is given in section 5 . In section 6 we have the embedding theorem i.e; construction of fuzzy graph $G$ from a given fuzzy graph $H$ such that $\langle C(G)>$ $\cong H$. In section 7 , based on this metric it is proved that an eccentric node of a fuzzy tree $G$ is a fuzzy end node of $G$ and a node is an eccentric node of a fuzzy tree if and only if it is a peripheral node of $G$ and the center of a fuzzy tree consists of either one or two neighboring nodes. In section 8 boundary node of a fuzzy graph based on strong sum distance is defined. Boundary nodes of fuzzy tree and complete fuzzy graph are discussed in this section. A complete node is defined and it is showed by an example that a complete node need not be a boundary node. Also an example to show that fuzzy cut node can be a boundary node is given in this section. Interior of a fuzzy graph based on strong sum distance is defined in Section 9. In a fuzzy graph there are nodes which are neither boundary nodes nor interior nodes. Interior node in complete fuzzy graph and boundary nodes in a cycle are also discussed in this section.

\section{Preliminaries}

A fuzzy graph(f-graph) (Mordeson and Nair 2000) is a triplet $G:(V, \sigma, \mu)$ where $V$ the vertex set, $\sigma$ is a fuzzy subset of $V$ and $\mu$ is a fuzzy relation on $\sigma$ such that $\mu(u, v)$ $\leq \sigma(u) \wedge \sigma(v) \forall u, v \in V$. We assume that $V$ is finite and non empty, $\mu$ is reflexive and symmetric. In all the examples $\sigma$ is chosen suitably. Also we denote the underlying crisp graph (Harary 1969) by $G^{*}:\left(\sigma^{*}, \mu^{*}\right)$ where $\sigma^{*}=\{u \in$ $V: \sigma(u)>0\}$ and $\mu^{*}=\{(u, v) \in V \times V: \mu(u, v)>0\}$. Here we assume $\sigma^{*}=V$. A fuzzy graph $H:(V, \tau, v)$ is called a partial fuzzy subgraph of $G:(V, \sigma, \mu)$ if $\tau(u) \leq \sigma(u) \forall u$ $\in \tau^{*}$ and $v(u, v) \leq \mu(u, v) \forall(u, v) \in v^{*}$. In particular we call $H:(V, \tau, \nu)$ a fuzzy subgraph of $G:(V, \sigma, \mu)$ if $\tau(u)$ $=\sigma(u) \forall u \in \tau^{*}$ and $v(u, v)=\mu(u, v) \forall(u, v) \in v^{*}$ and if in addition $\tau^{*}=\sigma^{*}$, then $H$ is called a spanning fuzzy subgraph of $G$. A weakest arc of $G:(V, \sigma, \mu)$ is an arc with least membership value. A path $P$ of length $n$ is a sequence of distinct nodes $u_{0}, u_{1}, \cdots, u_{n}$ such that $\mu\left(u_{i-1}, u_{i}\right)>0, i$ $=1,2,3, \cdots, n$ and the degree of membership of a weakest arc in the path is defined as its strength. If $u_{0}=u_{n}$ and $n \geq 3$, then $P$ is called a cycle and a cycle $P$ is called a fuzzy cycle(f-cycle) if it contains more than one weakest arc. A fuzzy graph $G:(V, \sigma, \mu)$ is a complete fuzzy graph (CFG) if $\mu(u, v)=\sigma(u) \wedge \sigma(v), \forall u, v \in \sigma^{*}$.

The strength of connectedness between two nodes $u$ and $v$ is defined as the maximum of the strengths of all paths between $u$ and $v$ and is denoted by $\operatorname{CONN}_{G}(u, v)$. A $u-v$ path $P$ is called a strongest $u-v$ path if its strength equals $\mathrm{CONN}_{G}(u, v)$. A fuzzy graph $G:(V, \sigma, \mu)$ is connected if for every $u, v$ in $\sigma^{*}, \operatorname{CONN}_{G}(u, v)>0$. Throughout this, we assume that $G$ is connected. An arc of a fuzzy graph is called strong if its weight is at least as great as the strength of connectedness of its end nodes when it is deleted and a $u-v$ path is called a strong path if it contains only strong arcs (Bhutani and Rosenfeld 2003a). If $\mu(u, v)>0$, then $u$ and $v$ are called neighbors. Also $v$ is called a strong neighbor if $\operatorname{arc}(u, v)$ is strong. The set of all neighbors of $u$ is denoted by $N(u)$ and the set of all strong neighbors of $u$ is denoted by $N_{s}(u)$. A node $u$ is a fuzzy end node of $G$ if it has exactly one strong neighbor in $G$. A strong path $P$ from $u$ to $v$ is a $u-v$ geodesic if there is no shorter strong path from $u$ to $v$ and the length of a $u-v$ geodesic is the geodesic distance from $u$ to $v$ denoted by $d_{g}(u, v)$ (Bhutani and Rosenfeld 2003b). Consider the fuzzy graphs $G_{1}:\left(V_{1}, \sigma_{1}, \mu_{1}\right)$ and $G_{2}:\left(V_{2}, \sigma_{2}, \mu_{2}\right)$ with $\sigma_{1}^{*}=V_{1}$ and $\sigma_{2}^{*}=V_{2}$. An isomorphism (Bhutani 1989) between two fuzzy graphs $G_{1}$ and $G_{2}$ is a bijective map $h: V_{1} \rightarrow V_{2}$ that satisfies $\sigma_{1}(u)=\sigma_{2}(h(u)) \forall u \in V_{1}$ and 
$\mu_{1}(u, v)=\mu_{2}(h(u), h(v)) \forall u, v \in V_{1}$ and is denoted by $G_{1} \cong G_{2}$.

An arc $(u, v)$ is a fuzzy bridge(f-bridge) of $G$ if deletion of $(u, v)$ reduces the strength of connectedness between some pair of nodes (Rosenfeld 1975). Equivalently, $(u, v)$ is a fuzzy bridge if and only if there exist $x, y$ such that $(u, v)$ is an arc on every strongest $x-y$ path. A node is a fuzzy cutnode (f-cutnode) of $G$ if removal of it reduces the strength of connectedness between some other pair of nodes (Rosenfeld 1975). Equivalently, $w$ is a fuzzy cutnode if and only if there exist $u, v$ distinct from $w$ such that $w$ is on every strongest $u-v$ path. A connected fuzzy graph $G:(V, \sigma, \mu)$ is a fuzzy tree (f-tree) if it has a spanning fuzzy subgraph $F:(V, \sigma, v)$, which is a tree, where for all arcs $(u, v)$ not in $F$ there exists a path from $u$ to $v$ in $F$ whose strength is more than $\mu(u, v)$. Thus for all arcs $(u, v)$ which are not in $F, \mu(u, v)<\operatorname{CONN}_{F}(u, v)$. A maximum spanning tree (MST) of a connected fuzzy graph $G:(V, \sigma, \mu)$ is a fuzzy spanning subgraph $T:(V, \sigma, v)$ such that $T^{*}$ is a tree and for which $\sum_{u \neq v} v(u, v)$ is maximum (Mordeson and Nair 2000). Note that for a fuzzy tree $G$, maximum spanning tree is unique and is the spanning fuzzy subgraph $F$ itself (Sunitha and Vijayakumar 1999). Depending on the $\operatorname{CONN}_{G}(u, v)$ of an arc $(u, v)$ in a fuzzy graph $G$, strong arcs are further classified as $\alpha-$ strong and $\beta$-strong and the remaining arcs are termed as $\delta$-arcs (Sunil and Sunitha 2009) as follows. Note that $G-(u, v)$ denotes the fuzzy subgraph of $G$ obtained by deleting the $\operatorname{arc}(u, v)$ from $G$. An arc $(u, v)$ in $G$ is called $\alpha$-strong if $\mu(u, v)>\operatorname{CONN}_{G-(u, v)}(u, v)$. An $\operatorname{arc}(u, v)$ in $G$ is called $\beta$-strong if $\mu(u, v)=C^{2} N_{G-(u, v)}(u, v)$. An $\operatorname{arc}(u, v)$ in $G$ is called a $\delta-\operatorname{arc}$ if $\mu(u, v)<\operatorname{CONN}_{G-(u, v)}(u, v)$. A $\delta-\operatorname{arc}$ $(u, v)$ is called a $\delta^{*}-\operatorname{arc}$ if $\mu(u, v)>\mu(x, y)$ where $(x, y)$ is a weakest arc of $G$.

\section{Strong sum distance in fuzzy graph}

Rosenfeld (1975) has defined $\mu$ - length of any $u-v$ path $P$ as the sum of reciprocals of arc weights in $P$ and distance between $u$ and $v$ called the $\mu$-distance denoted by $d_{\mu}(u, v)$, as the the smallest $\mu$ - length of $P$. Here we introduce a new definition for length of any $u-v$ path $P$ in a fuzzy graph $G$ and based on the new definition we introduce the concept of strong sum distance.

Definition 3.1. Let $G:(V, \sigma, \mu)$ be a connected fuzzy graph. For any path $P: u_{0}-u_{1}-u_{2}-u_{3}-\cdots \cdots-$ $u_{n}$, length of $P$ is defined as the sum of the weights of the arcs in $P$ i.e. $L(P)=\sum_{i=1}^{n} \mu\left(u_{i-1}, u_{i}\right)$. If $n=0$, define $L(P)$ $=0$ and for $n \geq 1, L(P)>0$. Also if $G$ is disconnected then $L(P)$ may be zero. For any two nodes $u, v$ in $G$, let $\boldsymbol{P}$ $=\left\{P_{i}: P_{i}\right.$ is a strong $u-v$ path $\left.i=1,2,3, \cdots\right\}$. The strong sum distance between $u$ and $v$ is defined as $d_{s s}(u, v)=$ Min $\left\{L\left(P_{i}\right): P_{i} \in \boldsymbol{P}, i=1,2,3, \cdots\right\}$.

Remark 3.2. If $\mu(u, v)=1 \forall(u, v) \in \mu^{*}$ then $d_{s s}(u, v)$ is the length of the shortest path as in crisp graph.

Theorem 3.3. In a fuzzy graph $G:(V, \sigma, \mu), d_{s s}: V \times$ $V \rightarrow[0,1]$ is a metric on V.i.e. $\forall u, v, w \in V$

(1) $d_{s s}(u, v) \geq 0 \forall u, v \in V$

(2) $d_{s s}(u, v)=0$ if and only if $u=v$

(3) $d_{s s}(u, v)=d_{s s}(v, u)$

(4) $d_{s s}(u, w) \leq d_{s s}(u, v)+d_{s s}(v, w)$

Proof. (1) and (2) follows from the definition. Next, since reversal of a strong path from $u$ to $v$ is a strong path from $v$ to $u$ and vice versa, $d_{s s}(u, v)=d_{s s}(v, u)$. Let $P_{1}$ be a strong $u-v$ path such that $d_{s s}(u, v)=L\left(P_{1}\right)$ and $P_{2}$ be a strong $v-w$ path such that $d_{s s}(v, w)=L\left(P_{2}\right)$. The strong path $P_{1}$ followed by strong path $P_{2}$ is a $u-w$ walk and since every walk contains one path, there exists a strong $u-w$ path in $G$ whose length is at most $d_{s s}(u, v)+d_{s s}(v, w)$.Therefore, $d_{s s}(u, w) \leq d_{s s}(u, v)+d_{s s}(v, w)$.

Definition 3.4. Let $G:(V, \sigma, \mu)$ be a connected fuzzy graph and let $u$ be a node of $G$. The eccentricity $e(u)$ of $u$ is the strong sum distance to a node farthest from $u$. Thus $e(u)=\max \left\{d_{s s}(u, v): v \in V\right\}$. For a node $u$, each node at strong sum distance $e(u)$ from $u$ is an eccentric node for $u$ denoted by $u^{*}$. $G$ is a unique eccentric node (u.e.n) fuzzy graph if each node in $G$ has a unique eccentric node. The radius $r(G)$ is the minimum eccentricity of the nodes, whereas the diameter $d(G)$ is the maximum eccentricity. A node $u$ is a central node if $e(u)=r(G)$, and $C(G)$ is the set of all central nodes. The fuzzy subgraph induced by $C(G)$ denoted by $<C(G)>=H:(V, \tau, v)$ is called the center of $G$. A connected fuzzy graph $G$ is self centered if each node is a central node i.e. $G \cong H$. A node $u$ is a peripheral node if $e(u)=d(G)$.

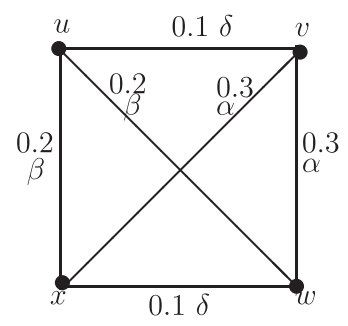

a)

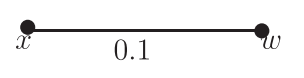

b)

Figure 1 Nodes in fuzzy graph $G$ based on strong sum distance. (a) Eccentric nodes, Central nodes, Peripheral nodes (b). Center of G.

Example 3.5. In Figure $1, d_{s s}(u, v)=0.5, d_{s s}(u, w)=0.2$, $d_{s s}(u, x)=0.2, d_{s s}(v, w)=0.3, d_{s s}(v, x)=0.3, d_{s s}(w, x)=0.4$. Therefore $e(u)=0.5, u^{*}=v, e(v)=0.5, v^{*}=u, e(w)=0.4$, $w^{*}=x, e(x)=0.4, x^{*}=w$. The central nodes are $w$ and $x$. The peripheral nodes are $u$ and $v$. Here $r(G)=0.4$ and $d(G)=0.5$. 
Remark 3.6. In crisp graph, a unique eccentric node graph is self centered if and only if each node of $G$ is eccentric. Note that the f-graph in Figure 1 is a unique eccentric node f-graph. In Figure 1, each node is eccentric and each node has a unique eccentric node but $G$ is not self centered.

Theorem 3.7. For any connected fuzzy graph $G$ : $(V, \sigma, \mu)$, the radius and diameter satisfy $r(G) \leq d(G) \leq$ $2 r(G)$.

Proof. $r(G) \leq d(G)$ follows from the definition of radius and diameter. Let $w$ be a central node of $G$. Therefore $e(w)$ $=r(G)$. Let $u$ and $v$ be two peripheral nodes of $G$. Therefore $e(u)=e(v)=d(G)$.

By triangle inequality $d_{s s}(u, v) \leq d_{s s}(u, w)+d_{s s}(w, v)$ i.e. $d(G) \leq r(G)+r(G) \cdot d(G) \leq 2 r(G)$. Therefore $r(G) \leq$ $d(G) \leq 2 r(G)$.

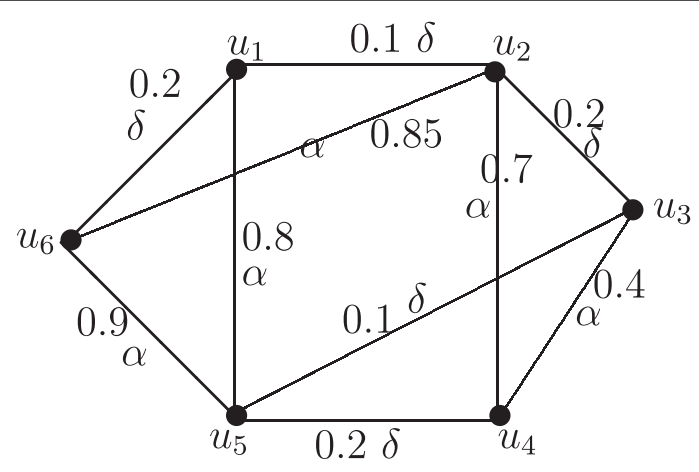

Figure 2 Fuzzy graph in which difference of eccentricities of the adjacent nodes is greater than 1 .

Remark 3.8. Note that in crisp graph, eccentricities of the adjacent nodes differ atmost by 1 . In Figure $2, u_{1}$ and $u_{2}$ are adjacent nodes. Note that $e\left(u_{1}\right)=3.65$ and $e\left(u_{2}\right)=$ 2.55 and hence $\left.\mid e\left(u_{1}\right)\right)-e\left(u_{2}\right) \mid=1.1$. But this result is true if $u$ and $v$ are strong neighbors as in following theorem.

Theorem 3.9. For every two strong neighbors $u$ and $v$ in a connected fuzzy graph $G:(V, \sigma, \mu),|e(u)-e(v)| \leq 1$.

Proof. Assume without loss of generality $e(u) \geq e(v)$. Let $x$ be a node farthest from $u$. i.e. $e(u)=d_{s s}(u, x) \leq d_{s s}(u, v)+$ $d_{s s}(v, x)$, by triangle inequality. Therefore $e(u) \leq d_{s s}(u, v)+$ $e(v)$, since $e(v) \geq d_{s s}(v, x)$. Since $u$ and $v$ are strong neighbors we have $d_{s s}(u, v) \leq 1$. Therefore $e(u) \leq 1+e(v) \Rightarrow 0$ $\leq e(u)-e(v) \leq 1$.

$\therefore|e(u)-e(v)| \leq 1$.

The above Theorem can be generalized as follows.

Theorem 3.10. For every two nodes $u$ and $v$ in a connected fuzzy graph $G:(V, \sigma, \mu),|e(u)-e(v)| \leq d_{s s}(u, v)$.
Proof. Assume without loss of generality $e(u) \geq e(v)$. Let $x$ be a node farthest from $u$. i.e. $e(u)=d_{s s}(u, x) \leq d_{s s}(u, v)+$ $d_{s s}(v, x)$, by triangle inequality. Therefore $e(u) \leq d_{s s}(u, v)+$ $e(v)$, since $e(v) \geq d_{s s}(v, x)$. i.e. $0 \leq e(u)-e(v) \leq d_{s s}(u, v)$.

Therefore $|e(u)-e(v)| \leq d_{s s}(u, v)$.

Remark 3.11. Note that in crisp graph, for every two adjacent nodes $u$ and $v,|d(u, x)-d(v, x)| \leq 1$. In Figure 2, $u_{1}$ and $u_{2}$ are adjacent nodes. Note that $d_{s s}\left(u_{1}, u_{3}\right)=3.65$ and $d_{s s}\left(u_{2}, u_{3}\right)=1.1$ and hence $\left.\mid d_{s s}\left(u_{1}, u_{3}\right)\right)-d_{s s}\left(u_{2}, u_{3}\right) \mid=$ 2.55. But this result is true if $u$ and $v$ are strong neighbors as in following theorem.

Theorem 3.12. For every two strong neighbors $u$ and $v$ in a connected fuzzy graph $G:(V, \sigma, \mu),\left|d_{s s}(u, x)-d_{s s}(\nu, x)\right|$ $\leq 1$ for every node $x$ of $G$.

Proof. Let $u$ and $v$ be strong neighbors in $G$ and let $x$ be any node of $G$. Assume $d_{s s}(u, x) \geq d_{s s}(v, x)$. Then by triangle inequality we have $d_{s s}(u, x) \leq d_{s s}(u, v)+d_{s s}(v, x)$. Since $u$ and $v$ are strong neighbors $d_{s s}(u, x) \leq 1+d_{s s}(v, x) \Rightarrow 0 \leq$ $d_{s s}(u, x)-d_{s s}(v, x) \leq 1$. Therefore $\left|d_{s s}(u, x)-d_{s s}(v, x)\right| \leq 1$.

Proposition 3.13. For any two real numbers $a, b$ such that $0<a \leq b \leq 2 a$, there exist a fuzzy graph $G$ such that $r(G)=a$ and $d(G)=b$.

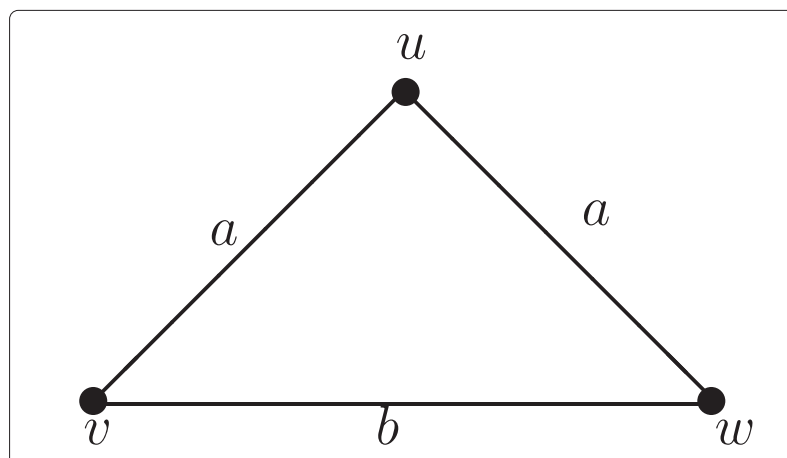

Figure 3 Fuzzy graph with all arcs strong.

Proof. From the definiton of strong arcs it follows that all arcs in Figure 3 are strong arcs. Therefore $d_{s s}(u, v)=a$, $d_{s s}(u, w)=a$ and $d_{s s}(v, w)=b$. Then $e(u)=a, e(v)=b$ and $e(w)=b$. Therefore $r(G)=a$ and $d(G)=b$.

Theorem 3.14. If $G:(V, \sigma, \mu)$ is a self centered fuzzy graph, then each node of $G$ is eccentric.

Proof. Assume $G$ is self centered and let $u$ be any node of $G$. Let $v$ be an eccentric node of $u$ i.e. $u^{*}=v$. Then $e(u)=d_{s s}(u, v)$. Since $G$ is self centered we have $e(v)=$ $e(u)$. Therefore $e(u)=d_{s s}(u, v)=e(v)$, which shows $u$ is an eccentric node of $v$ i.e. $v^{*}=u$. Hence the proof. 


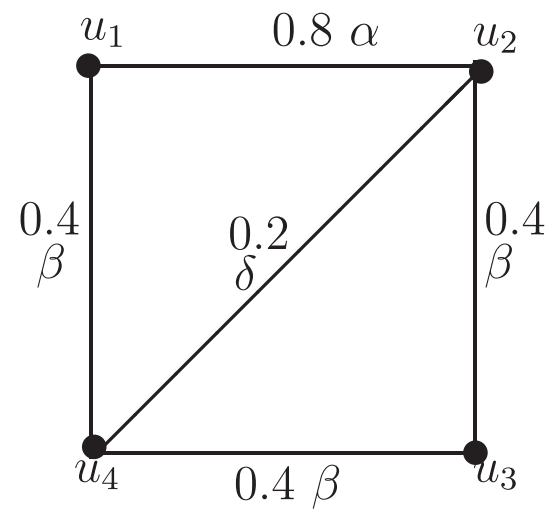

Figure 4 Self centered fuzzy graph.

Remark 3.15. Fuzzy graph $G:(V, \sigma, \mu)$ in Figure 4 is self centered with $e\left(u_{i}\right)=0.8, i=1,2,3,4$. The condition in Theorem 3.14 is not sufficient. In Figure 1, each node is eccentric but $G$ is not self centered.

Theorem 3.16. If $G:(V, \sigma, \mu)$ is a self centered fuzzy graph, then for every pair of nodes $u, v \in G, u \in V^{*}$ implies $v \in U^{*}$, where $U^{*}$ is the set of all eccentric nodes of $u$ and $V^{*}$ is the set of all eccentric nodes of $v$.

Proof. Assume $G$ is self centered and let $u, v$ be any two nodes of $G$. Let $u$ be an eccentric node of $v$. i.e. $d_{s s}(v, u)=$ $e(v)$, so we have $u \in V^{*}$. Now it is required to prove that $v$ $\in U^{*}$. Since $G$ is self centered we have $e(v)=e(u)$. Also we have $d_{s s}(v, u)=d_{s s}(u, v)=e(v)$. Therefore $e(u)=d_{s s}(u, v)$ which shows $v$ is an eccentric node of $u$ i.e. $v \in U^{*}$. Hence the proof.

Remark 3.17. The condition in Theorem 3.16 is not sufficient. In Figure 1, each node is eccentric and we have $u^{*}=v, v^{*}=u$ and $w^{*}=x, x^{*}=w$ but $G$ is not self centered.

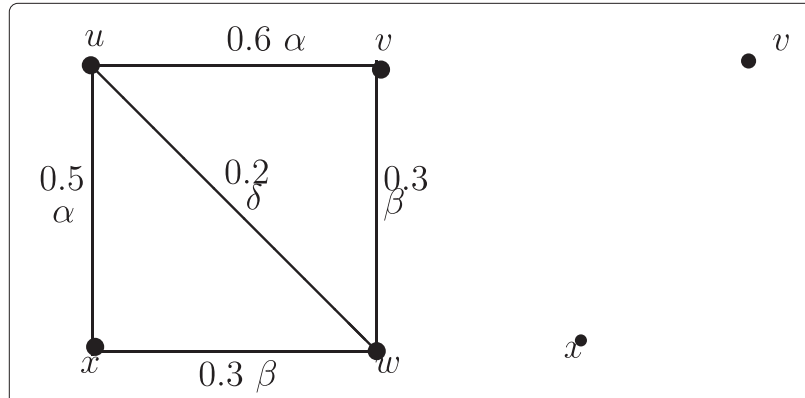

Figure 5 Fuzzy graph $G:(V, \sigma, \mu)<C(G)>$ with disconnected center.

Remark 3.18. The center of a connected fuzzy graph need be connected as shown in Figure 5.

Theorem 3.19. The center of every connected fuzzy graph $G:(V, \sigma, \mu)$ lies in a block of $G^{*}$.
Proof. Let $G:(V, \sigma, \mu)$ be a connected fuzzy graph. Assume that the center of $G$ does not lie in a block of $G^{*}$. Then there exist a node $v$ such that, $v$ is a cut node of $G^{*}$. Let $G_{1}$ and $G_{2}$ be any two components of $G^{*}-\nu$. Therefore each of $G_{1}$ and $G_{2}$ contain at leat one central node of $G$. Let $u$ be a node of $G$ such that $d_{s s}(u, v)=e(v)$. Let $P_{1}$ be a strong $u-v$ path such that $d_{s s}(u, v)=L\left(P_{1}\right)$, length of $P_{1}$. Then one of $G_{1}$ and $G_{2}$ contains no node in the path $P_{1}$, say $G_{2}$ contains no node of $P_{1}$. Let $w$ be a central node of $G$ that belongs to $G_{2}$ and let $P_{2}$ be a strong $v-w$ path such that $d_{s s}(v, w)=L\left(P_{2}\right)$, length of $P_{2}$. Therefore $d_{s s}(u, w)=$ $L\left(P_{1}\right)+L\left(P_{2}\right)$. Hence we have $e(w)>e(v)$, which contradicts $w$ is a central node of $G$. Hence center of every connected fuzzy graph $G:(V, \sigma, \mu)$ lies in a block of $G^{*}$.

Remark 3.20. As in crisp graphs, in fuzzy graphs every peripheral node is an eccentric node but not conversly. In Figure $1, u, v, w, x$ are eccentric nodes but $w$ and $x$ are not peripheral nodes.

Remark 3.21. In crsip graph no cutnode is a peripheral node but there are fuzzy graphs with peripheral nodes as fuzzy cut nodes. In Figure 1, nodes $u$ and $v$ are the peripheral nodes and node $v$ is a fuzzy cut node. Note that removal of the node $v$ reduces strength of connectedness between the nodes $w$ and $x$.

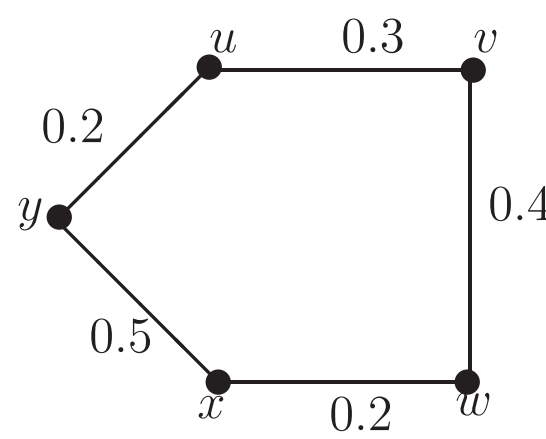

Figure 6 Fuzzy cycle which is not self centered.

Remark 3.22. A fuzzy cycle need not be self centered. In Figure $6, r(G)=0.6$ and $d(G)=0.7$ and the central node is $v$.

\section{Self centered fuzzy cycle}

Using the concept of $\mu$-eccentric nodes, in (Sunitha and Vijayakumar 1998) Sunitha and Vijayakumar has proved the sufficient conditions for a fuzzy graph $G$ such that $G^{*}$ is a cycle to be self centered. In this section, sufficient conditions for a cycle to be self centered based on strong sum distance are discussed. Note that all arcs in a fuzzy cycle are strong (Rosenfeld 1975).

Theorem 4.1. Let $G:(V, \sigma, \mu)$ be a fuzzy graph with $n$ nodes such that $G^{*} \cong C_{n}$, cycle on $n$ nodes with arcs $e_{i}=$ 
$\left(u_{i}, u_{i+1}\right) i=1,2, \cdots, n-1$ and $e_{n}=\left(u_{n}, u_{1}\right)$. Let $0<t<$ $s \leq 1$. Then $G$ is self centered if

1. $\mu\left(e_{i}\right)=t$ for $i=1,3,5, \cdots, n-1, \mu\left(e_{i}\right)=s$ for $i=2,4,6, \cdots, n-2$ and $\mu\left(e_{n}\right)=s$ when $n$ is even.

2. $\mu\left(e_{i}\right)=s$ for $i=1,3,5, \cdots, n-2, \mu\left(e_{i}\right)=t$ for $i=2,4,6, \cdots, n-1$ and $\mu\left(e_{n}\right)=s$ when $n$ is odd and $n=4 k-1$, where $k=1,2,3, \cdots$.

3. $\mu\left(e_{i}\right)=t$ for $i=1,3,5, \cdots, n-2, \mu\left(e_{i}\right)=s$ for $i=2,4,6, \cdots, n-1$ and $\mu\left(e_{n}\right)=t$ when $n$ is odd and $n=4 k+1$, where $k=1,2,3, \cdots$.

Also,

$r(G)=\left\{\begin{array}{l}k(t+s), n=4 k \text { or } n=4 k+1, k=1,2,3, \cdots \\ k(t+s)-t, n=4 k-1, k=1,2,3, \cdots \\ k(t+s)+t, n=4 k+2, k=1,2,3, \cdots\end{array}\right.$

Illustration 1. Take $t=0.3$ and $s=0.4$. Figures 7, 8, 9, $10,11,12,13$ and 14 illustrates the above theorem.

Case 1. $n$ is even and $n=4 k$ where $k=1,2, r\left(C_{4}\right)=0.7$ and $r\left(C_{8}\right)=1.4$.
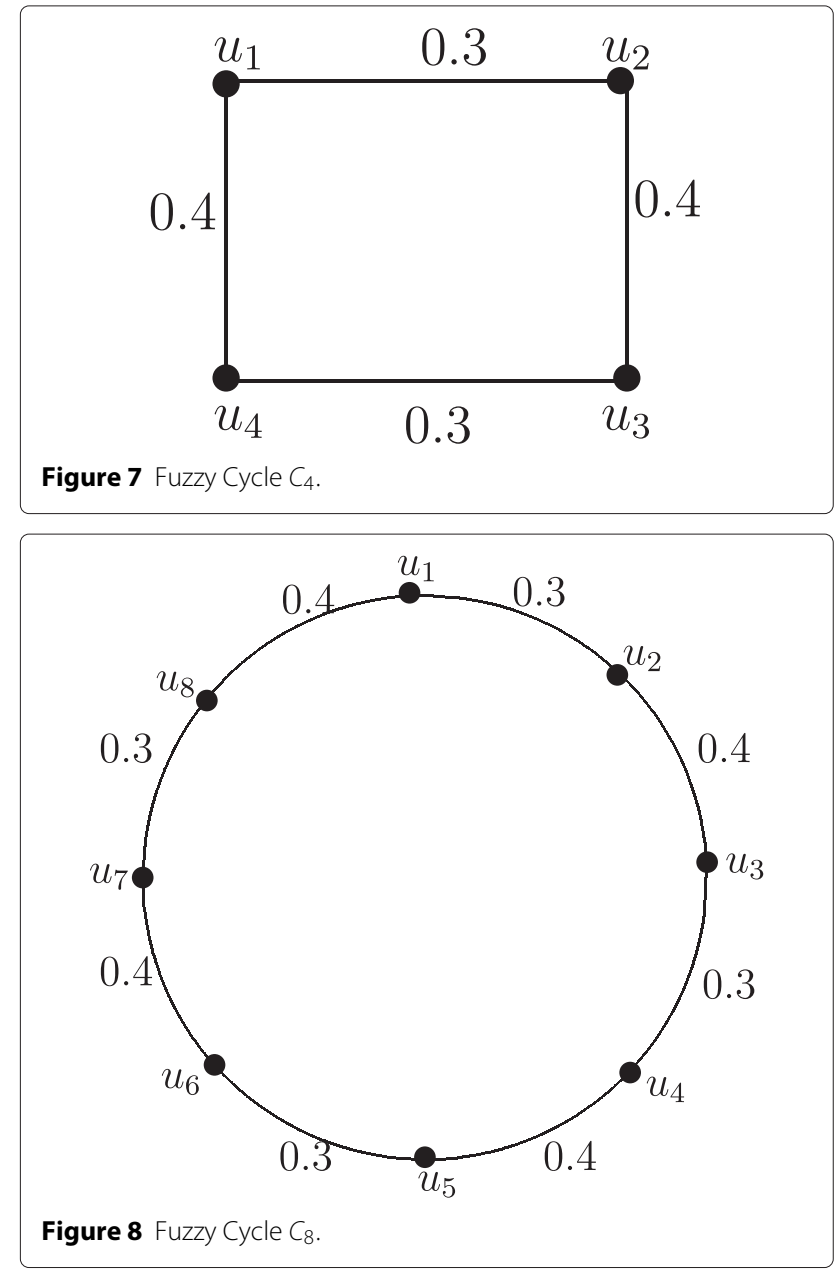

Case 2. $n$ is even and $n=4 k+2$ where $k=1,2, r\left(C_{6}\right)=$ 1.0 and $r\left(C_{10}\right)=1.7$.
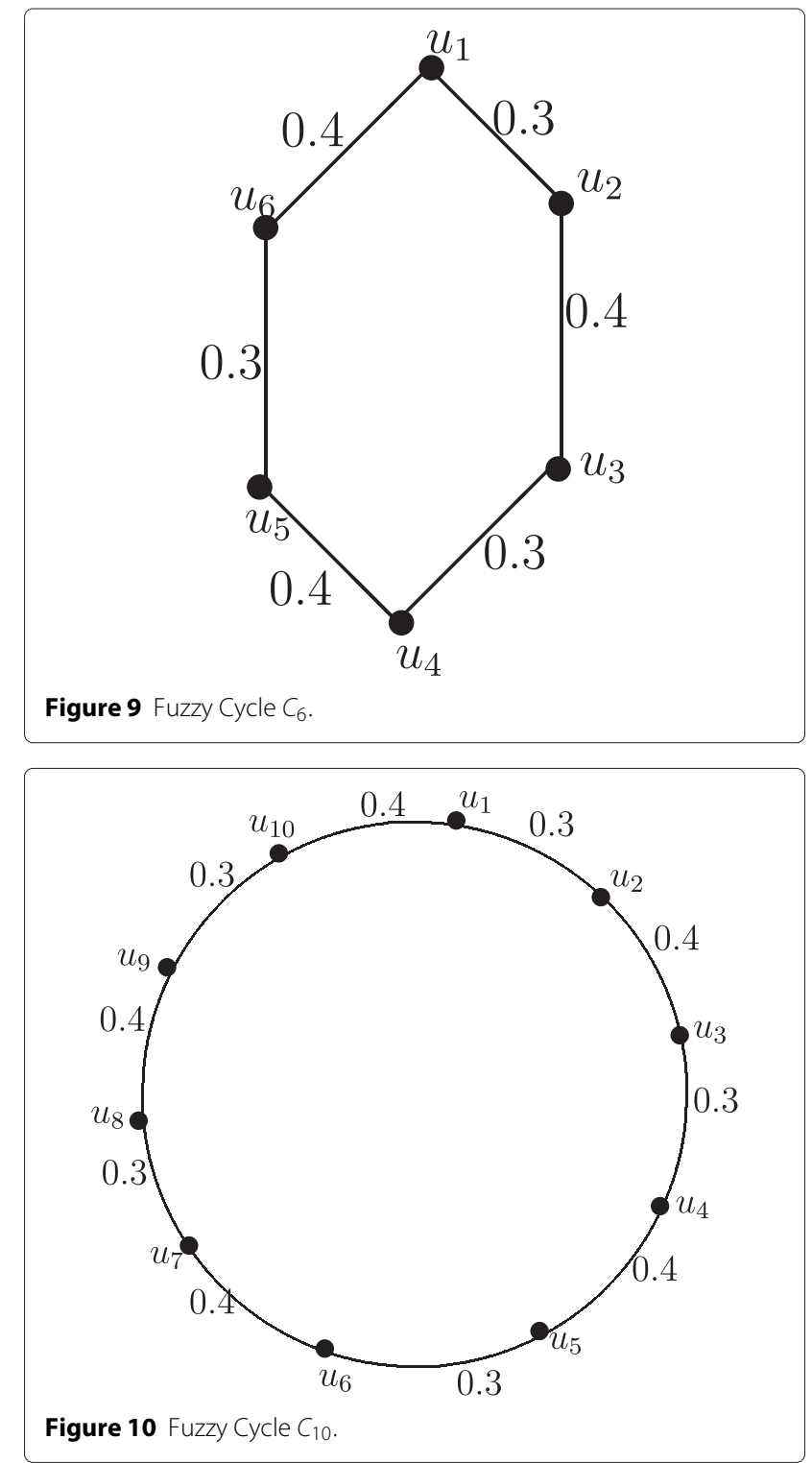

Case 3. $n$ is odd and $n=4 k-1$ where $k=1,2, r\left(C_{3}\right)=$ 0.4 and $r\left(C_{7}\right)=1.1$.

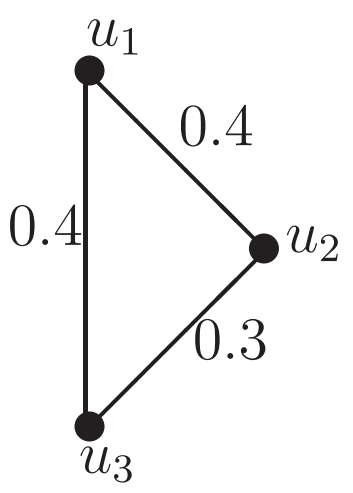

Figure 11 Fuzzy Cycle $C_{3}$. 


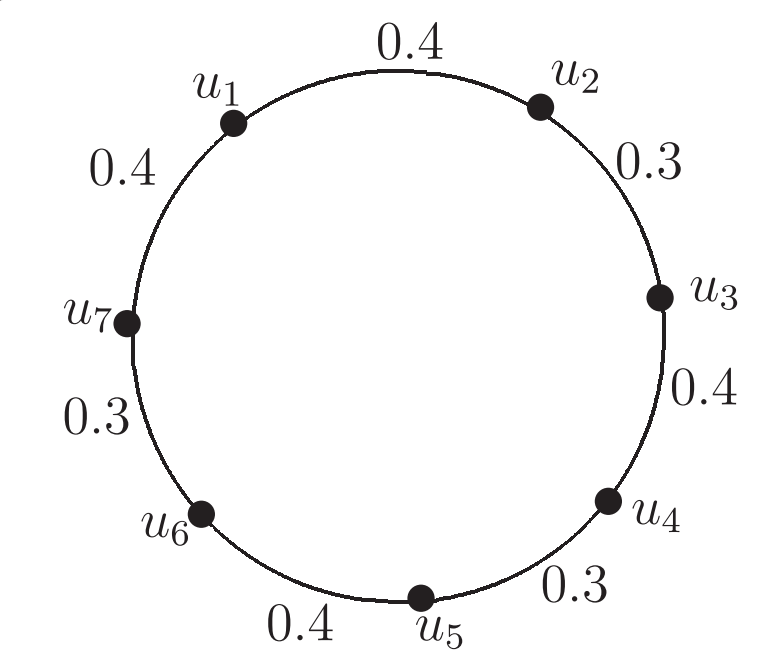

Figure 12 Fuzzy Cycle $C_{7}$.

Case 4. $n$ is odd and $n=4 k+1$ where $k=1,2, r\left(C_{5}\right)=$ 0.7 and $r\left(C_{9}\right)=1.4$.
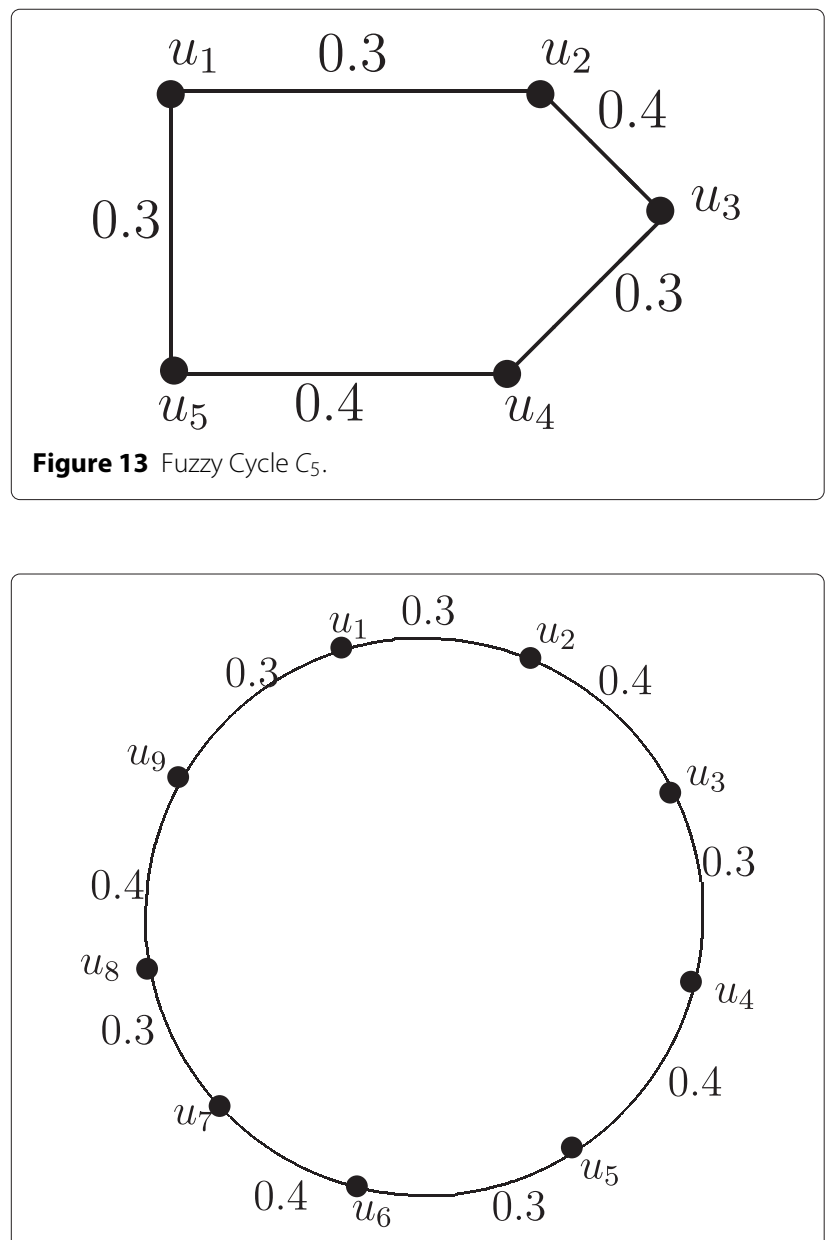

Figure 14 Fuzzy Cycle $C_{9}$.

\section{Strong sum distance in complete fuzzy graph}

In (Mini Tom Sunitha 2014) Mini and Sunitha proved that any $u-v$ path $P$ in a CFG is a strongest path if and only if either $u$ or $v$ is a weakest node in the path. In this section we first prove a necessary and sufficient condition for all paths in a CFG to be strongest and then a necessary and sufficient condition for a CFG to be self centered. Note that in a CFG all arcs are strong (Rosenfeld 1975; Bhutani and Rosenfeld 2003a).

Remark 5.1. A complete fuzzy graph need not be self centered. In Figure 15, $r(G)=0.3$ and $d(G)=0.5$ and the central node is $u$.

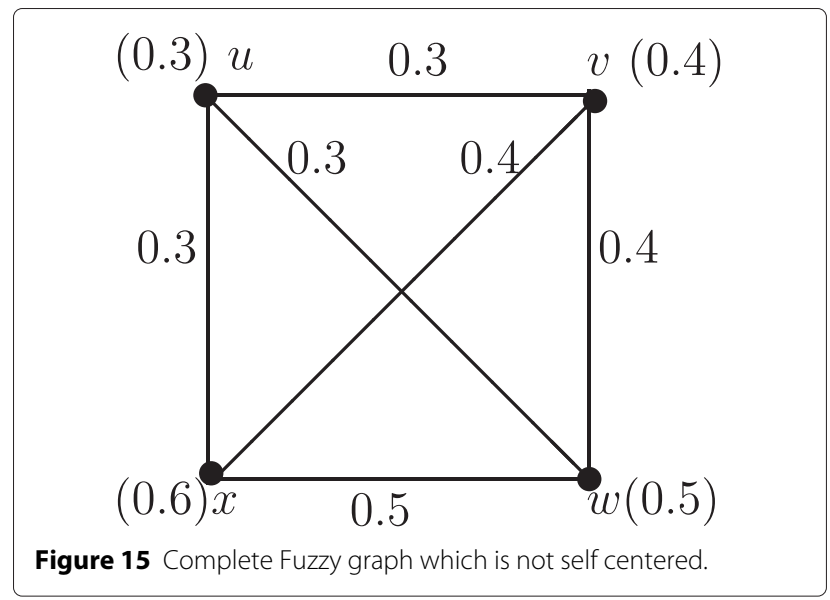

Theorem 5.2. Let $G:(V, \sigma, \mu)$ be a CFG with $\sigma^{*}$ $=\left\{u_{1}, u_{2}, u_{3}, \ldots, u_{n}\right\}$ such that $\sigma\left(u_{1}\right) \leq \sigma\left(u_{2}\right) \leq \sigma\left(u_{3}\right) \leq$ $\cdots \cdots \leq \sigma\left(u_{n}\right)$. Then the strong sum distance between any two nodes $u_{i}, u_{j}$ in $G$ is either $\mu\left(u_{i}, u_{j}\right)$ or $2 \sigma\left(u_{1}\right)$.

Proof. Let $u_{i}, u_{j}$ be any two nodes in $G$. We have $d_{s s}\left(u_{i}, u_{j}\right)=\min \left\{\mu\left(u_{i}, u_{j}\right), \mu\left(u_{i}, u_{k}\right)+\mu\left(u_{k}, u_{j}\right)\right\}$. Since $G$ is CFG we have $\mu\left(u_{i}, u_{k}\right)=\sigma\left(u_{i}\right) \wedge \sigma\left(u_{k}\right)$. Also since $\sigma\left(u_{1}\right)$ $\leq \sigma\left(u_{i}\right)$ for $i=2,3, \cdots, n$, when $k=1, \mu\left(u_{i}, u_{1}\right)=\sigma\left(u_{1}\right)$ and $\mu\left(u_{1}, u_{j}\right)=\sigma\left(u_{1}\right)$. Therefore $d_{s s}\left(u_{i}, u_{j}\right)=\min \left\{\mu\left(u_{i}, u_{j}\right)\right.$, $\left.2 \sigma\left(u_{1}\right)\right\}$.

Theorem 5.3. Let $G$ : $(V, \sigma, \mu)$ be a complete fuzzy graph on $n$ nodes, $n \geq 3$. All paths in $G$ are strongest paths if and only if there is at most one node $w$ in $G$ having different node strength and $\sigma(w)>\sigma\left(u_{i}\right) i=1,2,3, \cdots, n-1$.

Proof. Let $G:(V, \sigma, \mu)$ be a complete fuzzy graph on $n$ nodes, $\mathrm{n} \geq 3$, with $\sigma^{*}=\left\{u_{1}, u_{2}, u_{3}, \ldots, u_{n-2}, v, w\right\}$ and all nodes $u_{i}, i=1,2,3 \ldots, n-2$ have same node strength. Assume all paths in $G$ are strongest paths. Suppose $\sigma(v)$ $\neq \sigma(w) \neq \sigma\left(u_{i}\right), i=1,2,3, \cdots, n-2$.

Case 1: $\sigma\left(u_{i}\right)<\sigma(w)$ and $\sigma\left(u_{i}\right)<\sigma(v), i=1,2,3, \cdots, n-2$. Let $P: w-u_{1}-u_{2}-\ldots \ldots . .-u_{k}-v, k \leq n-2$, be a $w-v$ 
path. Then $P$ is not a strongest $w-v$ path since neither $w$ nor $v$ is a weakest node in $P$ (Mini Tom Sunitha 2014), contradiction.

Case 2: $\sigma\left(u_{i}\right)>\sigma(w)$ and $\sigma\left(u_{i}\right)>\sigma(v), i=1,2,3, \cdots, n-2$. Let $P$ be any $u_{i}-u_{j}$ path, $i, j=1,2,3, \cdots, n-2, i \neq j$ with either $v$ or $w$ as an internal node. Then $P$ is not a strongest $u_{i}-u_{j}$ path since neither $u_{i}$ nor $u_{j}$ is a weakest node in $P$ (Mini Tom Sunitha 2014), contradiction.

Case $3: \sigma\left(u_{i}\right)<\sigma(w)$ and $\sigma\left(u_{i}\right)>\sigma(v), i=1,2,3, \cdots, n-2$. Let $P$ be any $u_{i}-w$ path, $i=1,2,3, \cdots, n-2$, with $v$ as an internal node. Then $P$ is not a strongest $u_{i}-w$ path since neither $u_{i}$ nor $w$ is a weakest node in $P$ (Mini Tom Sunitha 2014), contradiction.

Hence there exist at most one node $w$ in $G$ having different node strength. Next to prove $\sigma(w)>\sigma\left(u_{i}\right) i$ $=1,2,3, \cdots, n-1$. Suppose not let, $\sigma(w)<\sigma\left(u_{i}\right) i=$ $1,2,3, \cdots, n-1$.Then by case 2 , we arrive at a contradiction. Hence $\sigma(w)>\sigma\left(u_{i}\right) i=1,2,3, \cdots, n-1$.

Conversely assume that there is at most one node $w$ in $G$ having different node strength and $\sigma(w)>\sigma\left(u_{i}\right) i=$ $1,2,3, \cdots, n-1$. Then any path $P$, joining any two nodes in $G$ is such that at least one of the end nodes of $P$ is a weakest node in the path $P$ and hence $P$ is a strongest path (Mini Tom Sunitha 2014).

Theorem 5.4. Let $G:(V, \sigma, \mu)$ be a CFG on $n$ nodes, $n$ $\geq 3$. Then $G$ is self centered if and only if all paths in $G$ are strongest paths.

Proof. Let $G:(V, \sigma, \mu)$ be a CFG. Assume $G$ is self centered. Then by Theorem 3.14, each node of $G$ is eccentric. Also for any two nodes $u, v$ in $G, e(u)=e(v)=r(G)=d(G)$. If possible assume that all paths in $G$ are not strongest paths. Therefore by Theorem 5.3, there exist at least two nodes $u, v$ with different node strength and let $w$ be an arbitrary node in $G$ such that $\sigma(w)$ is least. i.e. we have $\sigma(w)<\sigma(u)$ and $\sigma(w)<\sigma(v)$. Also we have $\mu(u, v)=$ $\sigma(u) \wedge \sigma(v)>\sigma(w)$ and $d_{s s}(u, v)=\operatorname{Min}\{\mu(u, v), 2 \sigma(w)\}$ by Theorem 5.2. Therefore $d_{s s}(u, v)>\sigma(w)$. Also we have $e(u)=\max \left\{d_{s s}(u, v): v \in V\right\}$.

Therefore $e(u)>\sigma(w) \ldots \ldots$. (1).

Now, for any node $u$ in $G$ we have $\mu(u, w)=\sigma(w)$ and therefore $d_{s s}(u, w)=\sigma(w)$ by Theorem 5.2.

Thus $e(w)=\max \left\{d_{s s}(w, u): u \in V\right\}=\sigma(w)$ ......... (2).

From (1) and (2) $e(u)>e(w)$, which contradicts our assumption that $G$ is self centered. Hence all paths in $G$ are strongest paths.

Conversely assume all paths in $G$ are strongest paths. Since all paths in $G$ are strongest paths, there is at most one node in $G$ having different strength and the strength of such a node is greater than the strength of all other nodes in $G$ by Theorem 5.3. Hence all arcs in $G$ have same strength . Also $d_{s s}(u, v)=\mu(u, v) \forall u, v$ by Theorem 5.2.
Hence for any two node $u, v$ in $G, e(u)=e(v)$. Therefore $G$ is self centered.

\section{Embedding theorem}

In this section, we shall consider the construction of a fuzzy graph $G$ from a fuzzy graph $H$ such that $<C(G)>$ $\cong H$.

Theorem 6.1. Let $H:\left(V, \sigma^{\prime}, \mu^{\prime}\right)$ be a fuzzy graph. Then there exists a connected fuzzy graph $G:(V, \sigma, \mu)$ such that $<C(G)>\cong H$.

Proof. Let $0<c=\wedge \sigma^{\prime}(u)$. Construct a fuzzy graph $G$ : $(V, \sigma, \mu)$ from $H$ as follows. Take four new nodes $u_{1}, u_{2}, v_{1}, v_{2}$ and put $\sigma^{*}=\sigma^{*} \cup\left\{u_{1}, u_{2}, v_{1}, v_{2}\right\}$ where $\sigma$ $=\sigma^{\prime}$ for all nodes $w$ in $H, \mu=\mu^{\prime}$ for all $\operatorname{arcs}(u, v)$ in $H$. Let $\sigma\left(u_{i}\right)=\sigma\left(v_{i}\right)=t(t \leq c), i=1,2 ; \mu\left(u_{1}, u_{2}\right)=\mu\left(v_{1}, v_{2}\right)$ $=t$ and $\mu\left(u_{2}, w\right)=\mu\left(v_{1}, w\right)=t \forall w \in H$. Then clearly $G:(V, \sigma, \mu)$ is a fuzzy graph and by definition of strong arcs, the $\operatorname{arcs}\left(u_{1}, u_{2}\right),\left(v_{1}, v_{2}\right),\left(u_{1}, w\right),\left(v_{1}, w\right) \forall w \in H$ are strong arcs. Also we have $e(w)=2 t \forall w \in H$ and $e\left(u_{1}\right)=$ $e\left(v_{1}\right)=3 t$ and $e\left(u_{2}\right)=e\left(v_{2}\right)=4 t$. Thus $<C(G)>\cong H$ and $r(G)=2 t$ and $d(G)=4 t$.
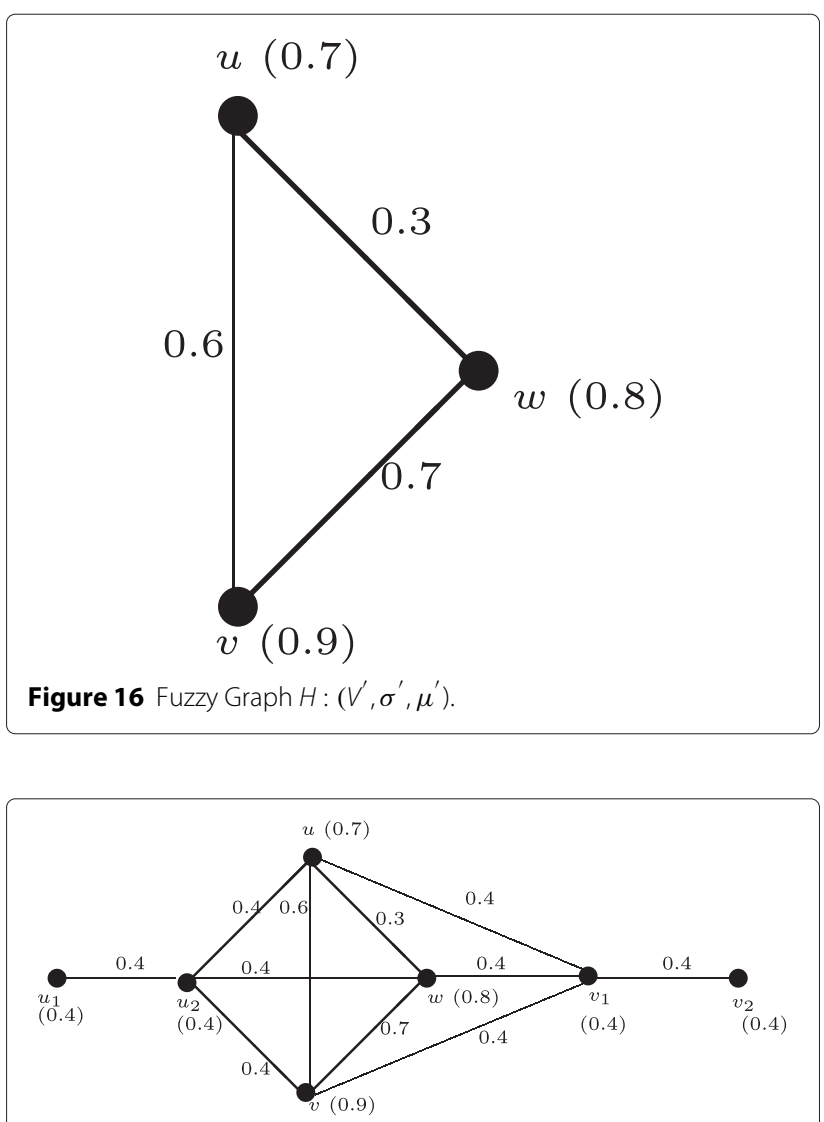

Figure 17 Fuzzy Graph $G:(V, \sigma, \mu)$ where Center $<C(G)>\cong H$. 
Example 6.2. Let $H:\left(V^{\prime}, \sigma^{\prime}, \mu^{\prime}\right)$ be a fuzzy graph (Figure 16) with $V^{\prime}=\{u, v, w\}$. Let $\sigma^{\prime}(u)=0.7, \sigma^{\prime}(v)=$ $0.9, \sigma^{\prime}(w)=0.8, \mu^{\prime}(u, v)=0.6, \mu^{\prime}(v, w)=0.7$ and $\mu^{\prime}(u, w)=0.3$. Construct the fuzzy graph $G:(V, \sigma, \mu)$ (Figure 17) from $H$ as follows. Take $t=0.4$. The node set of $G$ is $V=V^{\prime} \cup\left\{u_{1}, u_{2}, v_{1}, v_{2}\right\}$. Let $\sigma(u)=$ 0.7, $\sigma(v)=0.9, \sigma(w)=0.8, \sigma\left(u_{1}\right)=\sigma\left(u_{2}\right)=$ $\sigma\left(v_{1}\right)=\sigma\left(v_{2}\right)=0.4, \mu(u, v)=0.6, \mu(v, w)=0.7$, $\mu(u, w)=0.3, \mu\left(u_{1}, u_{2}\right)=0.4, \mu\left(v_{1}, v_{2}\right)=0.4, \mu\left(u_{2}, u\right)=$ $0.4, \mu\left(u_{2}, v\right)=0.4, \mu\left(u_{2}, w\right)=0.4, \mu\left(v_{1}, u\right)=0.4$, $\mu\left(v_{1}, v\right)=0.4, \mu\left(v_{1}, w\right)=0.4$. Note that $e(u)=e(v)=$ $e(w)=0.8$ and $e\left(u_{2}\right)=e\left(v_{1}\right)=1.2$ and $e\left(u_{1}\right)=$ $e\left(v_{2}\right)=1.6$. Thus $\langle C(G)>\cong H$ and $r(G)=0.8$ and $d(G)=1.6$.

\section{Strong sum distance in fuzzy trees}

Many metric properties of fuzzy trees are studied using $\mu$ - distance (Sunitha and Vijayakumar 1998), $g$ - distance (Sameena K and Sunitha 2011) and fuzzy detour $g$ - distance (Linda and Sunitha 2014a). In this section a similar study is carried out on fuzzy trees using strong sum distance. Note that an f-graph $G:(V, \sigma, \mu)$ is an $\mathrm{f}$-tree if and only if it has no $\beta$ strong arcs (Sunil and Sunitha 2009).

Theorem 7.1. Let $G:(V, \sigma, \mu)$ be a fuzzy tree and $F:(V, \sigma, v)$ be the maximum spanning tree of $G$. Then for each node u in $G, e(u)$ in $G$ is same as e $(u)$ in $F$.

Proof. Let $G:(V, \sigma, \mu)$ be a fuzzy tree and let $u$ be any arbitrary node in $G$. Let $e(u)=k$, i.e. $\exists$ a node $v$ in $G$ such that $d_{s s}(u, v)=k$, which implies that there is strong $u-v$ path $P$ in $G$ such that $L(P)=k$. Since $G$ is an $\mathrm{f}$-tree, $P$ is the unique strong $u-v$ path in $G$. Let $F:(V, \sigma, v)$ be the maximum spanning tree of $G$. Since $G$ is a fuzzy tree, $F$ is the unique maximum spanning tree of $G$ and contains all strong arcs of $G$. Thus $F$ contains the unique strong $u-v$ path and $d_{s s}(u, v)=k$. Hence $e(u)=k$ in $F$.

Corollary 7.2. Let $G:(V, \sigma, \mu)$ be a fuzzy tree and $F:(V, \sigma, v)$ be the maximum spanning tree of $G$. Then $<C(G)>\cong<C(F)>$ and center of a fuzzy tree consists of either one or two neighboring nodes.

Remark 7.3. Theorem 7.1 does not hold for a fuzzy graph which is not a fuzzy tree. The fuzzy graph $G$ in Figure 18 is not a fuzzy tree and hence $G$ has more than one maximum spanning tree. We have $e(u)=0.4$, $e(v)=0.4, e(w)=0.2$ and $e(x)=0.4$ in $G, e(u)=0.8$, $e(v)=1.1, e(w)=0.5$ and $e(x)=1.1$ in $F_{1}$ and $e(u)$ $=1, e(v)=1.1, e(w)=1.3$ and $e(x)=1.3$ in $F_{2}$. Note that the eccentricity of node $u$ is not same in $G, F_{1}$ and $F_{2}$.

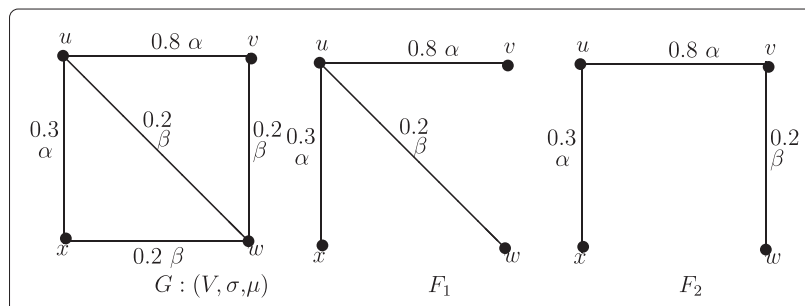

Figure 18 A fuzzy graph $G$ which is not an f-tree and its MST's $F_{1}$ and $F_{2}$.

Theorem 7.4. Let $G:(V, \sigma, \mu)$ be a fuzzy tree and $F$ : $(V, \sigma, v)$ be the maximum spanning tree of $G$. Then $G$ and $F$ have the same set of eccentric nodes.

Proof. Let $G:(V, \sigma, \mu)$ be a fuzzy tree and $F:(V, \sigma, v)$ be the maximum spanning tree of G. By Theorem 7.1, for any node $u$, eccentricity of $u$ in $G$ is same as eccentricity of $u$ in $F$. Let $e(u)=k$ and let $u^{*}=v$ in $G$. Then there is a strong $u-v$ path $P$ in $G$, which is also in $F$ such that $L(P)$ $=k$. Therefore $u^{*}=v$ in $F$ also. Similarly we can prove that for any node $u$ in $F$, if $u^{*}=v$ in $F$ then $u^{*}=v$ in $G$ also. Hence the proof.

Theorem 7.5. An eccentric node of a fuzzy tree $G$ : $(V, \sigma, \mu)$ is a fuzzy end node of $G$.

Proof. Let $G:(V, \sigma, \mu)$ be a fuzzy tree and let $u$ be an eccentric node of $G$. Then $u$ is an eccentric node of $F$, the maximum spanning tree of $G$ by Theorem 7.1. Since $F$ is a tree $u$ is an end node of $F$ and hence a fuzzy end node of $F$. Since $G$ and $F$ have the same set of fuzzy end nodes (Sameena K and Sunitha 2011), $u$ is fuzzy end node of $G$.

Remark 7.6. The converse of Theorem 7.5 does not hold as we see in Figure 19. In Figure 19, $x$ is a fuzzy end node. We have $u^{*}=w, v^{*}=w, w^{*}=u$ and $x^{*}=w$. Thus $x$ is not an eccentric node of $G$.

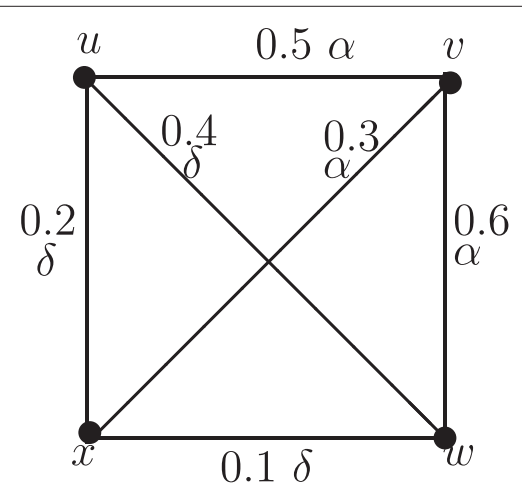

Figure 19 Fuzzy graph with fuzzy end node which is not eccentric node. 
Remark 7.7. Theorem 7.5 does not hold for a fuzzy graph which is not a fuzzy tree as we see in Figure 18. In Figure 18 , the eccentric nodes of $G$ are $u, v$ and $x$, but $G$ has no fuzzy end nodes.

Theorem 7.8. Let $G:(V, \sigma, \mu)$ be a fuzzy tree. Then a node $u$ of $G$ is an eccentric node if and only if $u$ is peripheral node of $G$.

Proof. Let $G:(V, \sigma, \mu)$ be a fuzzy tree and $u$, an eccentric node of $G$. Then by Theorem 7.5, $u$ is fuzzy end node of $G$. Since $G$ and $F$ have same set of fuzzy end nodes (Sameena K and Sunitha 2011), $u$ is fuzzy end node of $F$. Thus $u$ is an end node of $F$. Choose a fuzzy end node $v$ of $F$ other than $u$ such that $d_{s s}(u, v)$ in $F$ is maximum. Note that such a node $v$ exists since Theorem 2 of (Bhutani and Rosenfeld 2003c) sates that every fuzzy tree has at least two fuzzy end nodes. Then it follows that $d_{s s}(u, v)$ is the diameter of $F$ and hence the diameter of $G$. Therefore $u$ and $v$ are peripheral nodes of $G$.

Converse part follows from Remark 3.20.

Remark 7.9. From Theorem 7.5 and 7.8 we have the following Theorem.

Theorem 7.10. A peripheral node of a fuzzy tree $G$ : $(V, \sigma, \mu)$ is a fuzzy end node of $G$.

Remark 7.11. The converse of Theorem 7.10 is not true (Remark 7.6). Also note that Theorem 7.10 is true only in a fuzzy tree. In Figure 18, $u, v$ and $x$ are peripheral nodes, but $G$ has no fuzzy end nodes. Also in Figure 20, $v$ and $x$ are peripheral nodes of $G$, but $x$ is not a fuzzy end node of $G$.

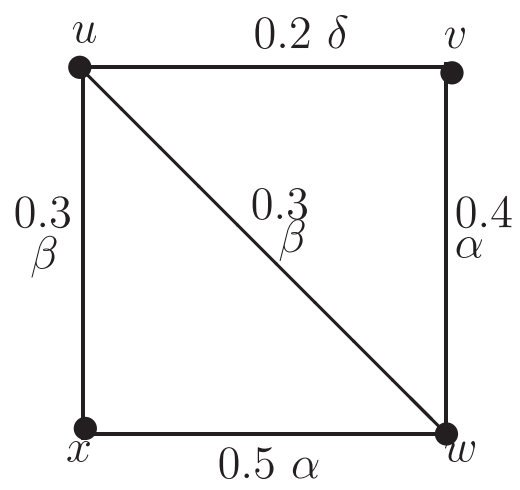

Figure 20 Fuzzy graph with peripheral node which is not fuzzy end node.

\section{Boundary node of a fuzzy graph}

Gary Chartrand and Ping Zang (2006) introduced the concepts of boundary vertex and boundary based on geodesic distance in crisp graph. Linda and Sunitha (2012) introduced the concepts of boundary node and boundary based on geodesic distance in fuzzy graphs. In this section we introduce the same concepts based on strong sum distance in fuzzy graphs. Also we define complete node based on strong sum distance and show by an example that a complete node need not be a boundary node.

Definition 8.1. A node $v$ in a connected fuzzy graph $G:(V, \sigma, \mu)$ is a boundary node of a node $u$ if $d_{s s}(u, v)$ $\geq d_{s s}(u, w)$ for each neighbor $w$ of $v$; while a node $v$ is a boundary node of a fuzzy graph $G$ if $v$ is a boundary node of some node of $G$.

The set of all boundary nodes of $u$ is denoted by $u^{b}$. The fuzzy subgraph induced by the boundary nodes of $G$ is called the boundary of $G$ denoted by $\partial(G)$.

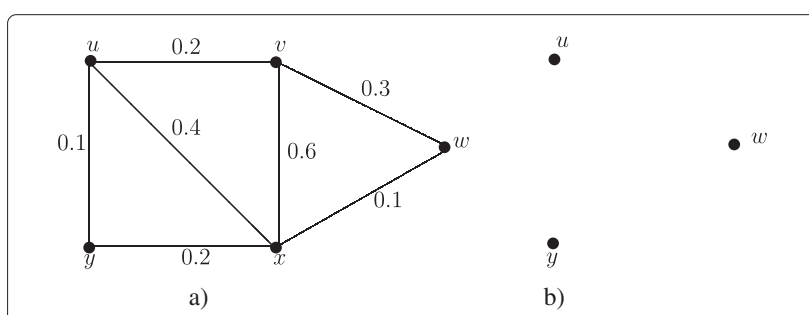

Figure 21 Boundary nodes in fuzzy Graph $G$ based on strong sum distance. (a). Boundary nodes (b). Boundary $\partial(G)$.

Example 8.2. Consider the fuzzy graph in Figure 21(a). Here $u^{b}=\{w, y\}, v^{b}=\{u\}, w^{b}=\{u\}, x^{b}=\{w\}, y^{b}=\{w\}$.

Hence the boundary nodes of $G$ are $u, w$ and $y$.

Remark 8.3. In a connected fuzzy graph every eccentric node is a boundary node, but a boundary node need not be an eccentric node. Consider the fuzzy graph in Figure 21. The eccentric nodes $u$ and $w$ are boundary nodes of $G$ but the boundary node $y$ is not an eccentric node of $G$.

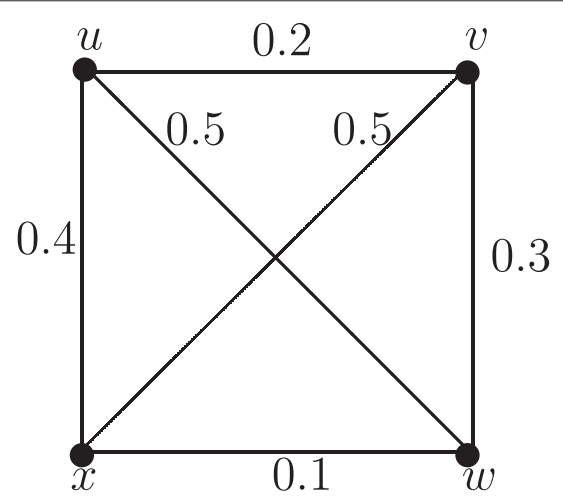

Figure $22 G:(V, \sigma, \mu)$ which is not self centered.

Example 8.4. In Figure 22, $e(u)=0.9$ and $u^{*}=v, e(v)=$ 1.4 and $v^{*}=w, e(w)=1.4$ and $w^{*}=v, e(x)=0.9$ and $x^{*}=$ 
$w$. Also $u^{b}=v, v^{b}=w, w^{b}=v, x^{b}=w$. Hence the nodes $v, w$ are peripheral nodes, eccentric nodes and boundary nodes. Note that $G$ is not self centered.

Definition 8.5. A node $v$ in a fuzzy graph $G$ is called a complete node if the fuzzy subgraph induced by the neighbors of $v$ form a complete fuzzy graph.

Remark 8.6. In crisp graph a vertex $v$ of $G$ is a boundary vertex of every vertex distinct from $v$ if and only if $v$ is a complete vertex of $G$ (Chartrand and Zang 2006) but in fuzzy graphs, based on strong sum distance a complete node need not be a boundary node as shown in Figure 23. Node $u$ is a complete node but it is not a boundary node. Also it may be noted that a node which is a boundary node of all other nodes need not be complete. Node $w$ is boundary node of all other nodes, but it is not complete.

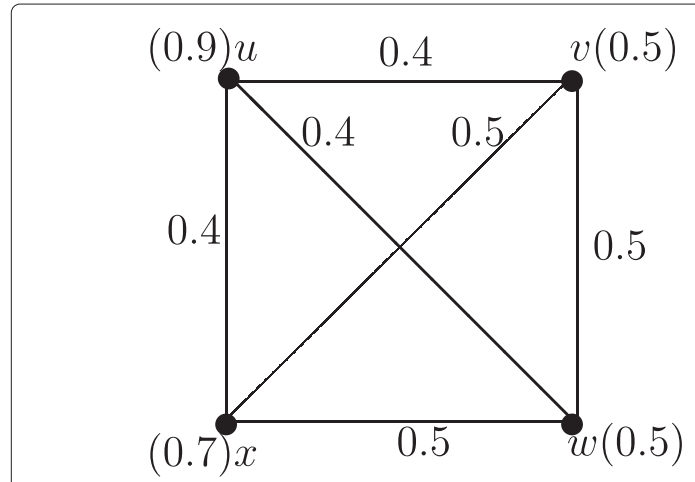

Figure 23 Complete node in a fuzzy graph.

Remark 8.7. In crisp graphs no cut node of a connected graph $G$ is a boundary node of $G$ (Chartrand and Zang 2006), but in fuzzy graphs which is not a fuzzy tree, a fuzzy cut node can be a boundary node as in Figure 24. In Figure 24, the boundary nodes of $G$ are $u, v$ and $x$. Note that node $u$ is a fuzzy cut node. Node $w$ is neither fuzzy cut node nor boundary node.

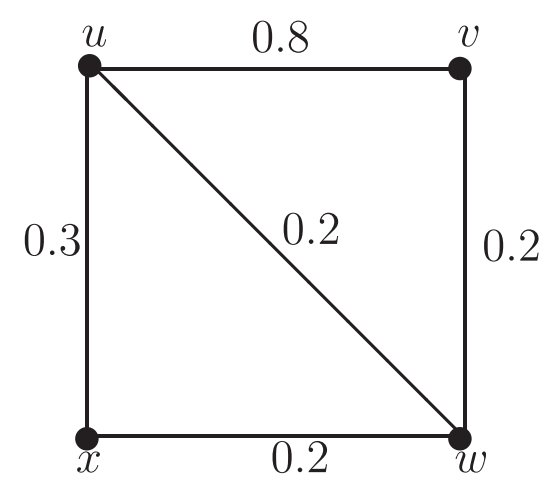

Figure 24 Fuzzy graph in which fuzzy cut node is a boundary node.
Theorem 8.8. In a fuzzy graph $G:(V, \sigma, \mu)$ a node $u$ is cut node implies $u$ is not a boundary node.

Proof. Let $G$ be a fuzzy graph. Assume, to the contrary that there exists a cut node $u$ of $G$ such that $u$ is a boundary node of some node $v$ of $G$. Let $G_{1}$ be the component of $G-u$ which contains $v$ and $G_{2}$ be any other component of $G-u$. If node $w$ is a neighbor of $u$ that belongs to $G_{2}$, then $d_{s s}(v, w)=d_{s s}(v, u)+x, 0<x \leq 1$, which contradicts our assumption that $u$ is a boundary node of $v$.

Theorem 8.9. In a fuzzy tree $G:(V, \sigma, \mu)$ no fuzzy cut node is a boundary node of $G$.

Proof. Let $G$ be a fuzzy tree. Assume, to the contrary that there exists a fuzzy cut node $u$ of $G$ such that $u$ is a boundary node of some node $v$ of $G$. Let $F$ be the unique maximum spanning tree of $G$. Theorem 8 of (Sunitha and Vijayakumar 1999) states that internal nodes of $F$ are fuzzy cut nodes of $G$. Hence $u$ is an internal node of $F$. By Theorem 7.4 and 7.5, $G$ and $F$ have the same set of eccentric nodes and an eccentric node of $G$ is a fuzzy end node of $G$. Now since $u$ is a boundary node it is an eccentric node of $G$ and hence $u$ is a fuzzy end node of $F$, which contradicts that $u$ is an internal node of $F$. Hence no fuzzy cut node of a fuzzy tree is a boundary node.

Theorem 8.10. In a fuzzy tree $G:(V, \sigma, \mu)$ a boundary node is a fuzzy end node.

Proof. Let $G$ be a fuzzy tree and $u$ is a boundary node of $G$. In a fuzzy tree every node is either a fuzzy cut node or a fuzzy end node (Bhutani and Rosenfeld 2003c). By Theorem 8.9 no fuzzy cut node is a boundary node of $G$. Hence $u$ is a fuzzy end node of $G$.

Remark 8.11. In a fuzzy tree $G:(V, \sigma, \mu)$ a fuzzy end node need not be a boundary node. In Figure 25(a), the boundary nodes are $v$ and $w$ and the fuzzy end nodes are $v$, $w$ and $y$. Note that the fuzzy end node $y$ is not a boundary node.

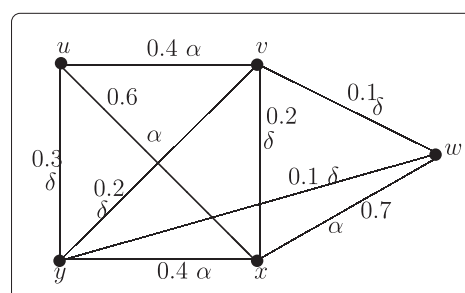

a)

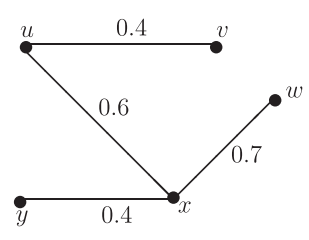

b)
Figure 25 Fuzzy end nodes and boundary nodes in fuzzy graph $G$ based on strong sum distance. (a). Fuzzy end node is not a boundary node (b). Unique maximum spanning tree. 
Theorem 8.12. Let $G:(V, \sigma, \mu)$ be a complete fuzzy graph on $n$ nodes, $n \geq 3$ and let $u_{0}$ be a node of G. Every node distinct from $u_{0}$ is a boundary node of $u_{0}$ if and only if $u_{0}$ is a weakest node of $G$.

Proof. $n \geq 3$. Let $u_{0}$ be a node of $G$ such that every node distinct from $u_{0}$ is a boundary node of $u_{0}$. i.e; we have $u_{0}^{b}=\left\{u_{i}, i=1,2,3, \ldots, n-1\right\}$. To prove that $u_{0}$ is a weakest node of $G$. Suppose not. Let $u_{k}$ be a node of $G$ such that $\sigma\left(u_{k}\right)$ is least. Then by definition of CFG, $\mu\left(u_{0}, u_{k}\right)=\sigma\left(u_{k}\right)$ and $\mu\left(u_{0}, u_{i}\right)=\sigma\left(u_{0}\right) \wedge \sigma\left(u_{i}\right), i \neq k$. Therefore we have $\mu\left(u_{0}, u_{k}\right)<\mu\left(u_{0}, u_{i}\right)$. Also, $d_{s s}\left(u_{0}, u_{k}\right)$ $=\mu\left(u_{0}, u_{k}\right)$ and $d_{s s}\left(u_{0}, u_{i}\right)=\min \left\{\mu\left(u_{0}, u_{i}\right), 2 \sigma\left(u_{k}\right)\right\}$, $i \neq k$ by Theorem 5.2. Clearly $d_{s s}\left(u_{0}, u_{k}\right)<$ $d_{s s}\left(u_{0}, u_{i}\right)$. Therefore by definition of boundary node $u_{k}$ is not a boundary node of $u_{0}$, which contradicts the assumption that every node distinct from $u_{0}$ is a boundary node of $u_{0}$. Therefore $u_{0}$ is a weakest node of $G$.

Conversly assume that $u_{0}$ is a weakest node of $G$. Then by definition of CFG, $\mu\left(u_{0}, u_{i}\right)=\sigma\left(u_{0}\right), i=$ $1,2,3, \ldots, n-1$. Also for any node $u_{i}$ of $G d_{s s}\left(u_{0}, u_{i}\right)$ $=\mu\left(u_{0}, u_{i}\right)$. Hence by definition of boundary node, every node distinct from $u_{0}$ is a boundary node of $u_{0}$.

Corollary 8.13. In a CFG $G:(V, \sigma, \mu)$, if $u_{0}$ is the unique weakest node then every node distinct from $u_{0}$ are boundary nodes of $G$, whereas if the weakest node is not unique then all the nodes of $G$ are boundary nodes of $G$.

\section{Interior node of a fuzzy graph}

Gary Chartrand and Ping Zang (2006) introduced the concepts of interior vertex and interior based on geodesic distance in crisp graph. Linda and Sunitha (2012) introduced the concepts of interior node and interior based on geodesic distance in fuzzy graphs. In this section we introduce the same concepts based on strong sum distance in fuzzy graph. In crisp graph the interior nodes are precisely those nodes that are not boundary nodes (Chartrand and Zang 2006).

Definition 9.1. Any node $w$ in a connected fuzzy graph $G:(V, \sigma, \mu)$ is said to lie between two other nodes $u$ and $v$ ( both different from $w$ ) with respect to strong sum distance if $d_{s s}(u, v)=d_{s s}(u, w)+d_{s s}(w, v)$.

Definition 9.2. A node $w$ is an interior node of a connected fuzzy graph $G:(V, \sigma, \mu)$ if for every node $u$ distinct from $w$, there exist a node $v$ such that $w$ lies between $u$ and $v$.

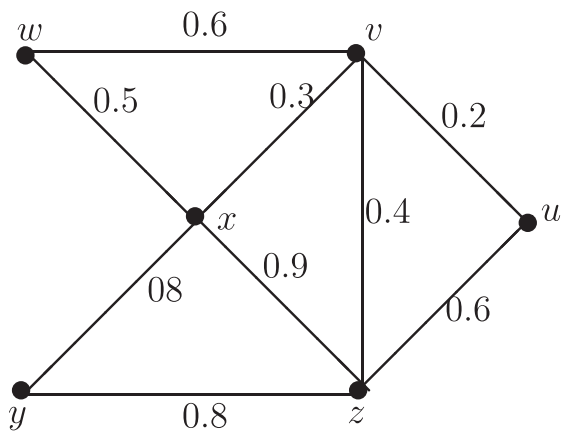

Figure 26 Fuzzy graph in which a node is neither an interior node nor a boundary node.

Example 9.3. Consider the fuzzy graph in Figure 26. The interior nodes are $x, w$ and $z$ and boundary nodes are $u$ and $v$. Node $y$ is neither a boundary node nor an interior node.

Theorem 9.4. Let $G:(V, \sigma, \mu)$ be a fuzzy graph. $A$ boundary node of $G$ is not an interior node of $G$.

Proof. Let $v$ be a boundary node of a connected fuzzy graph $G:(V, \sigma, \mu)$, say $v$ is a boundary node of the node $u$. Assume, to the contrary, that $v$ is an interior node of $G$. Therefore by definition of interior node, there exist a node $w$ distinct from $u$ and $v$ such that $v$ lies between $u$ and $w$. Let $P$ be that strong $u-w$ path in which $v$ lies between $u$ and $w$. i.e; $P=u-v_{1}-v_{2}-v_{3}-\ldots-v=v_{j}-v_{j+1}-\ldots .-v_{k}=w$, $1<j<k$. Now $v_{j+1} \in N(u)$ and $d_{s s}\left(u, v_{j+1}\right)=d_{s s}(u, v)+k$, $0<k \leq 1$, which contradicts that $v$ is a boundary node of $u$.

Remark 9.5. The converse of Theorem 9.4 is not always true. In fuzzy graph, all nodes which are not boundary nodes need not be interior nodes. In Figure 26, the boundary nodes are $u$ and $v$ and interior nodes are $x, w$ and $z$. Node $y$ is neither a boundary node nor an interior node.

Remark 9.6. In a complete fuzzy graph $G:(V, \sigma, \mu)$, by Theorem 8.12 and Corollary 8.13 there exists at most node which is not a boundary node. Therefore in a CFG there exist at most one node which in an interior node. The interior node if it exists is the unique weakest node of $G$.

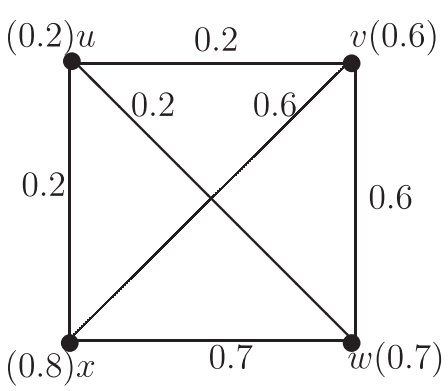

Figure 27 A complete fuzzy graph with an interior node. 
Example 9.7. In Figure 27, we have $d_{s s}(u, v)=0.2$, $d_{s s}(u, w)=0.2, d_{s s}(u, x)=0.2, d_{s s}(v, w)=0.4, d_{s s}(v, x)=0.4$, $d_{s s}(w, x)=0.4$ and $d_{s s}(v, w)=d_{s s}(v, u)+d_{s s}(u, w), d_{s s}(v, x)$ $=d_{s s}(v, u)+d_{s s}(u, x)$ and $d_{s s}(w, x)=d_{s s}(w, u)+d_{s s}(u, x)$. Hence $u$ is the only interior node. By Theorem 8.12 nodes $v, w, x$ are boundary nodes.

Theorem 9.8. In a fuzzy tree $G:(V, \sigma, \mu)$ a node is an interior node if and only if it is a fuzzy cut node.

Proof. Let $G$ be a fuzzy tree and $F$, the unique maximum spanning tree of $G$. Let $u$ be an interior node of $G$. Hence by Theorem $9.4 u$ is not a boundary node of $G$. Suppose $u$ is not a fuzzy cut node of $G$. Theorem 8 of (Sunitha and Vijayakumar 1999) states that internal nodes of $F$ are fuzzy cut nodes of $G$. Hence $u$ is not an internal node of $F$. $u$ is an end node of $F$ and hence a fuzzy end node of $G$. Thus $u$ does not lie between any two nodes of $G$ since there is only one strong arc incident on $u$. Therefore $u$ is not an interior node of $G$, which contradicts our assumption. Hence $u$ is a fuzzy cut node of $G$.

Conversely, let $u$ be a fuzzy cut node of $G$. Hence $u$ is an internal node of $F$. Since $F$ is a tree, for any node $w$ different $u$ there exist an end node $v$ of $F$ such that $d_{s s}(w, v)=d_{s s}(w, u)+d_{s s}(u, v)$. Thus $u$ is an interior node of $G$.

\section{Conclusion}

The idea of strong sum distance which is a metric, in a fuzzy graph is introduced. The concepts of eccentricity, radius, diameter, center, self centered f-graphs etc. are studied using this metric. A characterization of self centered complete fuzzy graph is obtained and conditions under which a fuzzy cycle is self centered are established. A necessary and sufficient condition for all paths in a CFG with $n \geq 3$ to be strongest paths is obtained. Also discussed the construction of a fuzzy graph $G$ from a given fuzzy graph $H$ such that $<C(G)>\cong H$. We have proved that based on this metric, an eccentric node of a fuzzy tree $G$ is a fuzzy end node of $G$ and a node is an eccentric node of a fuzzy tree if and only if it is a peripheral node of $G$ and the center of a fuzzy tree consists of either one or two neighboring nodes. The concepts of boundary nodes and interior nodes in a fuzzy graph based on strong sum distance are introduced. Some properties of boundary nodes, interior nodes and complete nodes are studied.

\section{Competing interests}

The authors declare that they have no competing interests.

\section{Authors' contributions}

The authors MT and SMS introduced the idea of strong sum distance in a fuzzy graph and studied various concepts in fuzzy graph based on this metric and drafted the manuscript. Both authors read and approved the final manuscript.

\section{Author details}

${ }^{1}$ Department of Mathematics, SCMS School Of Engineering and Technology, Karukutty, 683582 Kerala, India. ${ }^{2}$ Department of Mathematics, National Institute of Technology Calicut, Kozhikode, 673601 Kerala, India.

Received: 23 February 2015 Accepted: 19 March 2015

Published online: 06 May 2015

\section{References}

Abdul Jabbar N, Naoom JH, Ouda EH (2009) Fuzzy dual graph. J Al Nahrain Univ 12:168-171

Akram M (2011) Bipolar fuzzy graphs. Inform Sci 181:5548-5564

Akram M (2012) Interval-valued fuzzy line graphs. Neural Comput Appl 21:145-150

Akram M (2013) Bipolar fuzzy graphs with applications. Knowledge-Based Syst $39: 1-8$

Akram M, Alshehri N (2014) Intuitionistic fuzzy cycles and intuitionistic fuzzy trees. Sci World J 2014:11. Article ID 305836

Akram M, Dudek WA (2011) Interval-valued fuzzy graphs. Comput Math Appl 61:289-299

Akram M, Dudek WA (2012) Regular bipolar fuzzy graphs. Neural Comput Appl 21:197-205

Akram M, Karunambigai MG (2011) Metric in Bipolar Fuzzy Graphs. World Appl Sci J 14(12):1920-1927

Akram M, Karunambigai MG (2012) Some metric aspects of intuitionistic fuzzy graphs. World Appl Sci J 17(12):1789-1801

Alshehri N, Akram M (2013) Cayley bipolar fuzzy graphs. Sci World J 2013:8. Article ID 156786

Alshehri N, Akram M (2014) Intuitionistic fuzzy planar graphs. Discrete Dynamics Nat Soc 2014:9. Article ID 397823

Bhattacharya P (1987) Some remarks on fuzzy graphs. Pattern Recognit Lett 6:297-302

Bhutani KR (1989) On automorphisms of fuzzy graphs. Pattern Recognit Lett 9:159-162

Bhutani KR, Rosenfeld A (2003a) Strong arcs in fuzzy graphs. Inform Sci 152:319-322

Bhutani KR, Rosenfeld A (2003b) Geodesics in fuzzy graphs. Electron Notes Discrete Math 15:51-54

Bhutani KR, Rosenfeld A (2003c) Fuzzy end nodes in fuzzy graphs. Information Sci 152:323-326

Chartrand G, Zang P (2006) Introduction to Graph Theory. Tata McGraw Hill Edition, Delhi

Cohen JE (1968) Interval graphs and food webs: a finding and a problem. Document 17696-PR, RAND Corporation, Santa Monica, CA

Harary F (1969) Graph Theory. Addison-Wesley Publishing Company Inc, Boston

Linda JP, Sunitha MS (2013) $\mu$ - Geodetic Iteration Number and $\mu$-Geodetic Number of a Fuzzy Graph. Gen Math Notes 18:1-12

Linda JP, Sunitha MS (2012) On g-eccentric nodes g-boundary nodes and g-interior nodes of a fuzzy graph. Int Jr Math Sci Appl 2:697-707

Linda JP, Sunitha MS (2014a) Fuzzy detour g-center of a fuzzy graph. Ann Fuzzy Math Inform 7(2):219-228

Linda JP, Sunitha MS (2014b) Fuzzy detour g-interior nodes and Fuzzy detour g-boundary nodes of a fuzzy graph. J Intell Fuzzy Syst 27:435-442

Mini Tom Sunitha MS (2014) Notes on types of arcs in a fuzzy graph. J Uncertainty Math Sci:1-4

Mordeson JN, Nair PS (2000) Fuzzy Graphs and Fuzzy Hypergraphs. Physica Verlag, Heidelberg

Namboothiri NMM, Kumar VA, Ramachandran PT (2013) Cayley fuzzy graphs. Far East J Math Sci 73:1-15

Nagoorgani A, Umamaheswari J (2010) Fuzzy detour $\mu$ center in fuzzy graphs. Int J Algorithms Comput Math 3(2):57-63

Pal M, Rashmanlou H (2013) Irregular Interval - valued fuzzy graphs. Annals of Pure and Applied Mathematics 3(1):56-66

Pal A, Samanta S, Pal M (2013) Concept of fuzzy planar graphs. In: Proceedings of the Science and Information Conference, London, UK. pp 557-563

Pramanik T, Samanta S, Pal M (2014) Interval - valued fuzzy planar graphs. Int J Mach Learn Cyber. doi:10.1007/s13042-014-0284-7

Rashmanlou H, Pal M (2013) Isometry on Interval - valued fuzzy graphs. Int J Fuzzy Math Arch 3:28-35 
Rosenfeld A (1975) Fuzzy Graphs. In: Zadeh LA, Fu KS, Shimura M (eds). Fuzzy Sets and their Application to Cognitive and Decision Processes. Academic Press, New York. pp 77-95

Samanta S, Pal M (2013) Fuzzy k-competition graphs and p-competition fuzzy graphs. Fuzzy Inf Eng. doi:10.1007/s12543-013-0140-6

Samanta S, Pal A, Pal M (2014) New concepts of fuzzy planar graphs. Int J Adv Res Artif Intell 3(1):52-59

Samanta S, Akram M, Pal M (2015) m-Step fuzzy competition graphs. J Appl Math Comput 47:461-472

Sameena K, Sunitha MS (2008) A Characterisation of g-selfcentered Fuzzy Graphs. J Fuzzy Math 16:787-791

Sameena K, Sunitha MS (2011) On g- distance in fuzzy trees. J Fuzzy Math 19:787-791

Sunil M, Sunitha MS (2009) Types of arcs in a fuzzy graph. Inform Sci 179:1760-1768

Sunitha MS, Vijayakumar A (1998) Some Metric Aspects Of Fuzzy Graphs. In: Proceedings Of The Conference on Graph Connections. Cochin University of Science and Technology, Cochin. pp 111-114

Sunitha MS, Vijayakumar A (1999) A characterization of fuzzy trees. Inform Sci 113:293-300

Talebi AA, Hossein R (2013) Isomorphism on Interval - valued fuzzy graphs. Annals of Fuzzy Mathematics and Informatics 6(1):47-58

Yeh RT, Bang SY (1975) Fuzzy relations, fuzzy graphs and their application to clustering analysis. In: Zadeh LA, Fu KS, Shimura M (eds). Fuzzy sets and their Application to Cognitive and Decision Processes. Academic Press, New York. pp 125-149

\section{Submit your manuscript to a SpringerOpen ${ }^{\mathcal{O}}$ journal and benefit from:}

- Convenient online submission

- Rigorous peer review

- Immediate publication on acceptance

- Open access: articles freely available online

- High visibility within the field

- Retaining the copyright to your article

Submit your next manuscript at $\gg$ springeropen.com 